Personal knowledge of the chemistry of neodymium - the spectrum (absorption) I thought too complicated-and samarium brought me to the conclusion that also between those two elements an unknown element was missing, but all investigation by old methods was fruitless. Another reason may also be of interest. On arranging the true hydrides (in which the hydrogen is negative towards the positive metal) of the elements of the 8th series of the periodic system according to the order of their atomic weights, we find the following remarkable regularity of the composition of those peculiar compounds :

$\mathrm{CsH}_{1}, \mathrm{BaH}_{2}, \mathrm{LaH}_{3}, \mathrm{CH}_{4}, \mathrm{PrH}_{3}, \mathrm{NdH}_{2},\left(\mathrm{XH}_{1}\right), \mathrm{SmH}_{0}$. As samarium does not combine with hydrogen (Muthmann) there must exist between neodymium and samarium an unknown element which forms the hydride $\mathrm{XH}$-and this element is illinium. $\mathrm{My}$ speculation has not proved futile.

Bohemian University,

Bohuslav Brauner.

Prague, June I2.

\section{Identity of Herpetomonas papatasii and Leishmania tropica.}

THREE successful experiments have been recorded by us in which cutaneous leishmaniasis was transmitted to man by inoculation of Herpetomonas papatasii from naturally infected sandflies (Phlebotomus papatasii $q$ \&) (Annals of Trop. Med. and Parasitol., vol. 20, No. 2). Noguchi ("Action of Certain Biological Chemical and Physical Agents upon Cultures of Leishmania ; Some Observations on Plant and Insect Herpetomonads." International Conference on Health Problems in Tropical America, 1924) and Kligler ("The Cultural and Serological Relationship of Leishmania." Transact. Roy. Soc. Trop. Med. and Hyg., vol. I9, Nos. 5 and 6, I926) have introduced methods of preparing immune sera and distinguishing Leishmania tropica, L. donovani, and $L$. braziliense by agglutination. Agglutination and cross agglutination experiments with cultures from the three experimental lesions and three strains from naturally acquired lesions (two from Palestine and one from Baghdad) definitely proved the identity of the organisms from the experimental lesions with Leishmania tropica. Herpetomonas papatasii is, therefore, a synonym of Leishmania tropica, and the fact that Phlebotomus papatasii is a natural transmitter of cutaneous leishmaniasis is completely established, since in addition to the evidence of experimental transmission the Herpetomonas naturally occurring in the above insect is shown to be biologically identical with Leishmania tropica.

We hope to publish shortly complete details of our experiments elsewhere.

S. ADLER.

Microbiological Institute,

Hebrew University, Jerusalem.

\section{Pernicious Grafting.}

THE Madeira agriculturist will be glad of any enlightenment on a devitalising influence frequently experienced in grafting a vigorous wild peach or nectarine seedling with a scion from a cultivated example. The wild seedling germinates from any chance seedstone thrown upon our terraces and develops rapidly into a sturdy tree, almost evergreen in character, crowded in the late autumn with a wealth of fascinating flower before the deciduous annual foliage has left the branches bare, and yielding from early spring onwards an overwhelming crop of disappointing fruit.

If such a stock is cut across and grafted from a more worthy example in February, organic union is perfectly established and the new grafts speedily develop into sturdy ligneous growth which, by November, exhibits a promise of the vigorous output of leaf and flower customary in the wild seedling. But something now happens, and the tree, paralysed or devitalised, passes through the winter and spring months without a single leaf- or flower-bud breaking forth into activity.

Vitality is entirely suspended, and the tree is apparently poisoned by the introduction of an incompatible sap, although a modified life or mere existence may endure indefinitely. The removal of the intrusive grafts is unavailing, for the condition is all-pervading and not one of local obstruction.

Madeira is scarcely four days distant from the London markets, and it is of economic and commercial importance to remove any impediment to the almost perennial output of our sun-ripened produce.

Madeira, June 24 . Michael, Grabham.

\section{Organo-Metallic Compounds.}

AN X-ray investigation of the structure of the series of compounds carbon-, silicon-, germanium-, tin-, and lead-tetraphenyls, now almost completed, appears to indicate an interesting field of research in the organometallic compounds. It is proposed to extend the work to other series of these compounds so that after a time a systematic study may be made of the effects of varying (i.) the element, and (ii.) the groups. In order that this may be attempted, the writer appeals to chemists for the loan of small quantities of any related organo-metallic compounds which may be stable solids in air at ordinary temperatures.

To give an idea of the quantity of material sufficient for this work, it may be stated that the crystal of germanium tetraphenyl kindly lent by Prof. G. T. Morgan and found to give satisfactory results measured only I. $5 \mathrm{~mm}$. by $[0.065 \mathrm{~mm} \text {. }]^{2}$, whilst from $0.15 \mathrm{gm}$. of powdered tin tetraphenyl kindly lent by Mr. A. E. Goddard, Mr. W. B. Saville, who is co-operating with me in this work, was able to grow a number of crystals suitable for use on both the photographic and the ionisation $\mathrm{X}$-ray spectrometers.

Davy Faraday Laboratory

20 Albemarle Street, London, W.I.

\section{Blood Reactions and Sex.}

THE method elaborated by Manoilov for distinguishing the sexes has been used by us in the case of the fowl. Male can certainly be distinguished from female by this test. Seven birds which previously functioned as hens but now, having undergone complete sex-reversal, are fecund and potent cocks, all exhibit the female reaction indubitably. This being so, this test may be expected to provide valuable corroborative evidence concerning the genetic sex of certain kinds of sexually abnormal individuals.

The reaction in the case of blood from a fowl from which the gonadic tissue has been removed is, in our hands, not yet sufficiently definite.

Animal Breeding Research Dept.,

F. A. E. CREW.

University of Edinburgh, July 5 . 